\title{
Correction: FTSJ1 regulates tRNA 2'-O- methyladenosine modification and suppresses the malignancy of NSCLC via inhibiting DRAM1 expression
}

Qihan He, Lin Yang, Kaiping Gao, Peikun Ding, Qianqian Chen, Juan Xiong, Wenhan Yang, Yi Song, Liang Wang, Yejun Wang, Lijuan Ling, Weiming Wu, Jisong Yan, Peng Zou, Yuhuan Chen and Rihong Zhai (D)

Correction to: Cell Death and Disease https://doi.org/10.1038/s41419-020-2525-x published online 11 May 2020

The original version of this article contained an error in the spelling of the author Yuchen Chen, which was incorrectly given as Yuhuan Chen. This has now been corrected in both the PDF and HTML versions of the article.

Published online: 03 June 2020 